\title{
Network Clustering for Voltage Control in Active Distribution Network Including Energy Storage Systems
}

\author{
Maryam Bahramipanah, Mostafa Nick, Rachid Cherkaoui and Mario Paolone \\ Ecole Polytechnique Fédérale de Lausanne \\ Lausanne, Switzerland
}

\begin{abstract}
This paper presents a network partitioning strategy for the optimal voltage control of Active Distribution Networks (ADNs) by means of Dispersed Energy Storage Systems (DESSs). The proposed partitioning is based on the concept of voltage sensitivity coefficients and is adopted for a decentralized voltagecontrol strategy specifically developed for radial ADNs. The aim of the partitioning is to decompose the network into quasiautonomous areas and to limit the information exchange only at the interfacing nodes between adjacent areas. The information exchange relies on Thévenin equivalents to represent the external grids of each cluster/area. The effectiveness of the proposed decentralized control approach is assessed with respect to an equivalent centralized control approach. Such an assessment is carried out using a numerical example referring to IEEE 123 buses distribution test feeder suitably adapted to include stochastic generation and DESSs.
\end{abstract}

Index Terms-Active distribution networks, distributed control, voltage control, network clustering, Thévenin equivalent model.

\section{INTRODUCTION}

As known, the increasing penetration of distributed energy resources into ADNs brings various planning and operation challenges fundamentally caused by the lack of direct control over stochastic and non-stochastic Distributed Generation (DG). Within this context, one of the most promising near-term solutions is represented by the indirect control of the network quality-of-service (e.g., node voltages) by means of Distributed Energy Storage Systems (DESSs). In this context, the question arises whether centralized controls are still the most appropriate to keep under control the ADN quality-of-service. A possible solution is to move from the centralized operation paradigm to the decentralized one by subdividing the network in quasiautonomous entities defined as areas, zones or clusters (these terms will be used as synonyms in the rest of the paper). It is considered that: (i) the operating information within each zone is not shared with the others and that (ii) each area is independently controlled by an own controller. This would inherently relax the computation burden associated to the central information processing by reducing the size of the searching space for control strategies and, as a consequence, enhance the ADNs operation security. In this respect, the paper

This work was performed at EPFL Distributed Electrical Systems Laboratory in the context of a research project sponsored by EOS Holding funds and entitled "Advanced control of distribution networks with the integration of dispersed energy storage systems". presents method to subdivide the distribution system grid into several zones according to voltage sensitivity coefficients criteria. It is assumed that each zone does not contain any directly-controllable generator, or load, except one controllable DESS (therefore, the number of DESS defines the number of independently-controlled zones). For sake of space, in this study the decentralized control refers only to the voltage one. Nevertheless, its extension to manage line congestion is straightforward.

In order to have almost independent operating zones, each one should have an overview of its neighbors without knowing their internal state. For this purpose, an algorithm is proposed to perform the online tracing of the Thévenin equivalent circuits seen from the common boundary bus of each pair of adjacent zones/areas. The algorithm follows continuously the changes of the system states. As it will be shown later, it gives a consistent result with reference to the equivalent centralized control.

The remainder of the paper is organized as follows: section II first summarizes the methods proposed in the literature for the network partitioning and, then, explains the one proposed in this contribution. Section III presents a distributed control method applied in the clustered network. Finally, in section IV, the validation of the proposed clustering methodology is presented by making reference to the IEEE 123 buses test case. Finally, the proposed decentralized control method is compared with its corresponding equivalent centralized one in order to demonstrate its effectiveness.

\section{NETWORK CLUSTERING APPROACHES}

The power network partitioning can be approached in several ways. A collection of zones of variable size has been proposed in [1] and [2] where supply/demand are kept balanced in order to efficiently control dispatchable DGs. The normal fluctuations in supply/demand (such as peak/off-peak load variations) change the boundaries of the zones. The drawback of this method is that all the buses need to register in all possible areas and also the proposed method requires the direct control of DGs with consequent scalability burdens. Reference [3] has proposed a partitioning algorithm using community detection theory as a super set of graph partitioning. It is assumed that the network is decomposed into different pre- 
defined areas and the proposed algorithm tries to merge them to keep the local reactive power balance. In this approach, the voltage is controlled only by adjusting the reactive power neglecting the fact that voltage control in distribution networks depends on both active/reactive powers as a function of the $R / X$ ratio of lines longitudinal parameters. In the context of transmission networks, the reactive power partitioning method has been presented in [4].

\section{A. Proposed clustering method}

In order to decouple the voltage control, given a set $B$ of DESSs optimally located in a balanced and radial ADN (for instance, following the procedure proposed in [5]), the proposed partitioning algorithm decomposes the ADN into several quasiautonomous zones; each under the control of one DESS. Therefore, the boundaries of each area are determined based on the following rule: each DESS within a zone of unknown extension must have significant influence on the co-zonal controlled variables (in our case the node voltages) in comparison with the nodes outside the zone of interest. In order to achieve such a goal, there is the need to quantify the influence of control variables (i.e., nodal power injection/absorptions of each DESS) on the controlled ones, namely the node voltages. Voltage Sensitivity Coefficients $(V S C)$ are used for this purpose (e.g., [6]). As known, VSC depends on the topology and the operating state of the grid. In our study we rely on the method presented in [7] to compute the $V S C s$. Given a certain operating state of the network, the VSC of node $i$ on node $j\left(V S C_{i \rightarrow j}\right.$ ) shows the change on voltage at node $j$ for a variation of the injected/absorbed active/reactive powers at node $i$. In this study, VSCs specifically link node voltages with respect to DESSs active/reactive power variations.

The steps for the decomposition of the network are described hereafter:

1) Computation of the VSC sensitivity matrices: for a given network operationg state $\Gamma$, two sensitivity matrices for both active and reactive powers $\left(\left[V S C^{P}\right]_{B \times N}\right.$ and $\left.\left[V S C^{Q}\right]_{B \times N}\right)$ are computed whose elements can be defined as in (1) where $N$ is the set of all network nodes. As known, VSC are formally defined as:

$V S C_{i \rightarrow j}^{P}=\left.\frac{\partial V_{j}}{\partial P_{i}}\right|_{\Gamma} ; V S C_{i \rightarrow j}^{Q}=\left.\frac{\partial V_{j}}{\partial Q_{i}}\right|_{\Gamma} ; i \in B ; j \in N$

2) Find DESSs voltage influence factors (VIF): the VIF of DESS located at node $i$ on node $j$ is defined as a the voltage change at node $j$ divided by the sum of the voltage changes at all the nodes of the network, caused by the active/reactive power output change of that DESS. As a result, two influence matrices $\left(\left[V I F^{P}\right]_{B \times N}\right.$ and $\left.\left[V I F^{Q}\right]_{B \times N}\right)$ are derived as in (2).

$$
V I F_{i \rightarrow j}^{P}=\left.\frac{\frac{\partial V_{j}}{\partial P_{i}}}{\sum_{n=1}^{N} \frac{\partial V_{n}}{\partial P_{i}}}\right|_{\Gamma}, V I F_{i \rightarrow j}^{Q}=\left.\frac{\frac{\partial V_{j}}{\partial Q_{i}}}{\sum_{n=1}^{N} \frac{\partial V_{n}}{\partial Q_{i}}}\right|_{\Gamma}
$$

3) Merge matrices $\left[V I F^{P}\right]_{B \times N}$ and $\left[V I F^{Q}\right]_{B \times N}$ : only one matrix $[V I F]_{B \times N}$ is defined to consider the effect of both active and reactive powers simultaneously. A linear relationship is built based on the concept of the electric distances using (3).
$[V I F]_{B \times N}=[\alpha]_{B \times N} \cdot\left[V I F^{P}\right]_{B \times N}+[\beta]_{B \times N} \cdot\left[V I F^{P}\right]_{B \times N}$

Each element of $[\alpha]_{B \times N}$ and $[\beta]_{B \times N}$ are defined as in (4).

$\alpha_{i \rightarrow j}=\frac{\sum R_{i j}}{\sqrt{\left(\sum R_{i j}\right)^{2}+\left(\sum X_{i j}\right)^{2}}} ; \quad \beta_{i \rightarrow j}=\frac{\sum X_{i j}}{\sqrt{\left(\sum R_{i j}\right)^{2}+\left(\sum X_{i j}\right)^{2}}}$
Where $\sum R_{i j}$ and $\sum X_{i j}$ are respectively the sum of the line
resistances/reactances of the path between node $i$ and node $j$

列

4) Assign each node to one DESS: as anticipated, in the scope of this paper, we consider only one DESS per area. For each node, the DESS that has the most impact is determined by finding the maximum component value of each column of the matrix $[V I F]_{B \times N}$ given by (3). More precisely, each node is assigned to the related area associated to the DESS with the highest VIF on that node. In this case, we can guarantee that each node belongs to one and only one area.

\section{DISTRIBUTED CONTROL BASED ON THE PROPOSED CLUSTERING}

In this section we describe a distributed control method for ADNs that considers the network clustering presented in section II. The entire power network is divided into $K$ areas as the result of the clustering. As stated earlier, each area includes only one DESS. Therefore, $K$ is equal to the total number of areas and DESSs as well.

\section{A. Common Boundary Bus $(C B B)$ between two adjacent areas}

Due to the hypothesis of the radial structure of the considered ADNs and the partitioning features, there is only one connection line between any two adjacent areas. A simple representation of a clustered network is shown in Fig. 1. As it can be seen, an area may have more than one neighbor. Each area is supposed to share no information about its internal state (i.e., node voltages) with the others except at the boundary buses. Indeed, as required by the control method presented later, one of the boundary state variables must be shared between each pair of adjacent areas. In this respect, for a given pair of adjacent areas $\left(A_{h}\right.$ and $A_{g}$ ), one of them is extended to include the boundary node of the other. In Fig. 1, area $A_{h}$ is extended to include bus $g$ of area $A_{g}$, which is one of its neighbors. Node $g$ is defined as the Common Boundary Bus $\left(C B B^{A_{h, g}}\right)$ of both areas $A_{h}$ and $A_{g}$. As a result, between any two adjacent areas one node is selected as $\mathrm{CBB}$. Obviously, the number of CBBs involved in a given area is equal to the number of its neighbors.

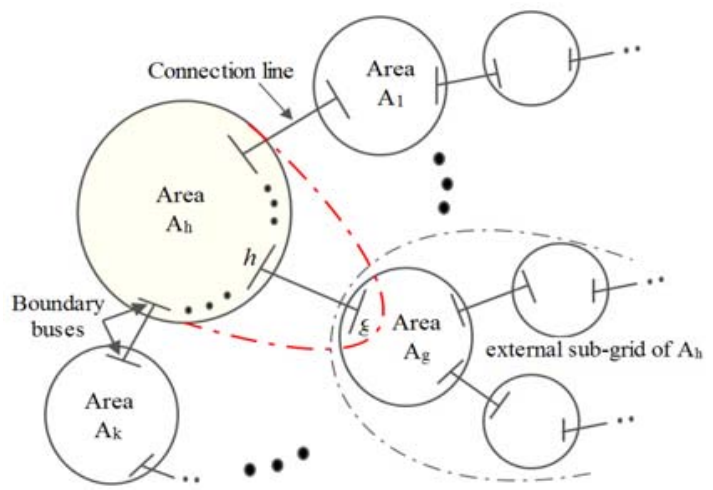

Figure 1. Representation of area $A_{h}$ with its $m$ neighbors. 


\section{B. Proposed distributed control thechnique}

The general framework of the proposed distributed control method relies on two tasks: 1) Distributed Local Control (DLC); 2) Exchange and update of CBBs information and Convergence condition check (E\&C).

\section{1) DLC task}

DLC task is performed for each area locally, independently and in a parallel manner. In this respect, it needs an equivalent representation of the external sub-grids connected to the area under study. For this purpose, the DLC relies on an online tracing of the Thévenin equivalent models of the abovementioned sub-grids as presented later (E\&C task). Another aim of this task is to define the voltage reference assigned to each slack node presented in each cluster. This is done only once by a centralized load flow in correspondence of the controller initialization (i.e., at $\mathrm{t}=0$ ).

Considering area $A_{h}$ (Fig. 1) the DLC task includes 5 steps:

Step 1: compute equivalent injection powers at all CBBs of area $A_{h}\left(\bar{S}_{C B B}^{A_{h, l}}\right)$

The equivalent complex power flowing from any adjacent area $A_{l}(l=1,2, \ldots, m)$ to $A_{h}\left(\bar{S}_{C B B}^{A_{h, l}}\right)$ through $C B B^{A_{h, l}}$ is calculated using active/reactive power flow equations, $m$ is the number of the neighbors of area $A_{h}$. At each time step, in the first iteration, an initial value is selected for each $\bar{S}_{C B B}^{A_{h, l}}$ (i.e., the initial value of the load/generation at this node before the control action). In the subsequent iterations, this complex power is updated thanks to the voltage values resulting from step 5 and the CBBs voltages update provided by the E\&C task.

Step 2: perform distributed power flow (DLF) independently and locally for area $A_{h}$

In this step, a load flow is performed locally for area $A_{h}$ considering $\bar{S}_{C B B}^{A_{h, l}}$ at the related CBBs calculated in step 1.

\section{Step 3: compute VSCs for area $A_{h}$}

VSCs are computed using the method presented in [7] for both active/reactive powers of DESS located in area $A_{h}$.

Step 4: compute equivalent Thévenin impedances for the external sub-grids including area $A_{h}$

The Thévenin impedances seen from the CBBs of area $A_{h}$ and toward area $A_{h}$ can be calculated as (5).

$$
\bar{Z}_{t h}^{A_{h, l}}=\frac{\left|\bar{V}_{C B B}^{A_{h, l}}\right|^{2}}{\bar{S}_{C B B}^{* A_{h, l}}} ; l=1, \ldots, m
$$

\section{Step 5: local control for area $A_{h}$ using VSCS}

In this step a simple control method based on VSCs is used to keep the voltage close to the desired value at each node belonging to $A_{h}$. Assuming just only one DESS in area $A_{h}$, this storage device is referred as the control center for this area. Consequently, the control variables of the optimization problems are the injected/absorbed active/reactive power of the DESS on the AC-side of the power converter (i.e., $P_{D E S S}^{A_{h}}$ and $\left.Q_{D E S S}^{A_{h}}\right)$.
The energy state-of-charge ( $S o C^{A_{h}}$ ) of $D E S S^{A_{h}}$ is simply defined as the remaining energy inside the device assuming, as a first approximation, a unity efficiency of the device. The energy flow is assumed to be positive during the DESS discharge and negative during its charge.

$S o C^{A_{h}}=S o C^{p r e, A_{h}} \pm P_{D E S S}^{A_{h}} \cdot \Delta t$

where $S o C^{p r e, A_{h}}$ is the $S o C$ in the previous time step and $\Delta t$ is the control time interval.

The objective function and the constraints of the control optimization problem is formulated for each area as (7).

$$
\begin{array}{ll}
\min _{\substack{P_{D E S S}^{A_{h}}, P_{D E S S}^{A_{h}} \\
\text { s.t }}} \sum_{d, t}\left\|\left|\bar{V}_{d, t}^{A_{h}}\right|-V_{\text {ref }}^{A_{h}}\right\| \\
\\
S_{O} C_{\min }^{A_{h}} \leq S o C^{A_{h}} \leq S o C_{\max }^{A_{h}} \\
\sqrt{P_{D E S S}^{A_{h}}{ }^{2}+Q_{D E S S}^{A_{h}}{ }^{2}} \leq S_{r}^{D E S S^{A_{h}}}
\end{array}
$$

Where $\left|\bar{V}_{d, t}^{A_{h}}\right|$ is the voltage magnitude of node $d$ in area $A_{h}$ at time $t\left(d=1,2, \ldots, N^{*}\right) . N^{*}$ is the number of the internal buses in $A_{h}$ including CBBs, and $V_{\text {ref }}^{A_{h}}$ is the voltage reference value.

The constraints for the optimization problem are related to the $S o C^{A_{h}}$ as well as the power converters ( $P Q$ capability curve of the converters). $S_{r}^{D E S S^{A_{h}}}$ is the rated power of the converter of DESS in area $A_{h}$. The storage devices are not allowed to be charged or discharged completely. If the $S o C^{A_{h}}$ limits are reached and the storage asked for further charge or discharge, the DESS should refuse to participate in delivering or absorbing power. If the limits are not reached yet, the normal operation is taken into account. These limits $\left(S o C_{\min }^{A_{h}}\right.$ and $\left.S o C_{\max }^{A_{h}}\right)$ are, in general, defined by the DESS manufacturer in order to prevent excessive discharge rates that would damage the storage system and/or reduce its life.

The voltage at bus $d$ could be expressed as its initial value plus a variation provided by the DESS.

$\left|\bar{V}_{d, t}^{A_{h}}\right|=\left|\bar{V}_{d, t}^{A_{h}, \text { init }}\right|+\Delta\left|\bar{V}_{d, t}^{A_{h}}\right|$

Where $\bar{V}_{d, t}^{A_{h}, \text { init }}$ comes from step 2. $\Delta\left|\bar{V}_{d, t}^{A_{h}}\right|$ is computed by the following approximated equation:

$\Delta\left|\bar{V}_{d, t}^{A_{h}}\right|=V S C_{b \rightarrow d}^{P} \cdot P_{D E S S}^{A_{h}}+V S C_{b \rightarrow d}^{Q} \cdot Q_{D E S S}^{A_{h}}$

where $b$ is related to the node where DESS is located in area $A_{h} . \quad V S C_{b \rightarrow d}^{P}$ and $V S C_{b \rightarrow d}^{Q}$ are the network node voltages sensitivity coefficients related to the DESS active and reactive power variations in area $A_{h}$.

At the end of the DLC task, the new voltages values for the internal buses and CBBs of $A_{h}$ are provided.

\section{2) $E \& C$ task}

In order to comply with the results we can derive from a centralized control, the following condition must be fulfilled. For each pair of adjacent areas, the voltage at the shared CBB must be unique. In other words, for a given $C B B^{A_{h, g}}$ linking $A_{h}$ 
and $A_{g}$, the equality constraint (10) must be respected; where the voltages $\bar{V}_{C B B}^{A_{h, g}}$ and $\bar{V}_{C B B}^{A_{g, h}}$ are computed separately for $A_{h}$ and $A_{g}$ respectively according to the DLC task.

$\bar{V}_{C B B}^{A_{h, g}}=\bar{V}_{C B B}^{A_{g, h}}$

The E\&C task first checks for all CBBs in the network whether (10) is simultaneously satisfied. If this is the case, then the distributed control at the current time $t$ converged and the active and reactive power setups of all the DESSs are updated according to the last results provided at step 5 of DLC. If it is not the case, the E\&C task forces the voltage at each $\mathrm{CBB}$ to be unique according to the procedure described below. Then, we return to step 1 of DLC task for each area to perform a new iteration.

The update of the CBBs voltages relies on the following assumption: for a given pair of adjacent areas $A_{h}$ and $A_{g}$, the corresponding $C B B^{A_{h, g}}$ divides the whole network in two distinct sub-grids. These sub-grids contain $A_{h}$ and $A_{g}$ respectively. Each of them is assumed to be represented by a Thévenin equivalent model seen from $C B B^{A_{h, g}}$ (step 4 of DLC). These equivalent circuits are connected each other as shown in Fig. 2.

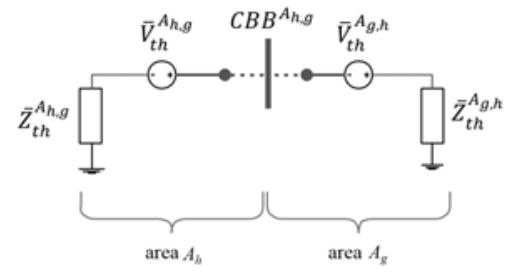

Figure 2. Thévenin equivalent circuit models seen from $C B B^{A_{h, g}}$

From this equivalent model, the unique voltage at $C B B^{A_{h, g}}$ is calculated by (11).

$$
\bar{V}_{C B, \text { new }}^{A_{h, g}}=\bar{V}_{C B B, \text { new }}^{A_{g, h}}=\frac{\frac{\bar{V}_{C B B}^{A_{h, g}}}{\bar{Z}_{t h}^{A_{h, g}}}+\frac{\bar{V}_{C B B}^{A_{g, h}}}{\bar{Z}_{t h}^{A_{g, h}}}}{\frac{1}{\bar{Z}_{t h}^{A_{h, g}}}+\frac{1}{\bar{Z}_{t h}^{A_{g, h}}}}
$$

The main feature of the proposed method is the independence of the load flow calculation for each area and the possibility for any area to join into or move back from the control scheme.

\section{CASE STUdy}

This section demonstrates the benefits of the partitioning method using a case study that refers to the IEEE 123 nodes distribution test feeder. The schematic of this network is shown in Fig. 3. It is supposed to involve non-dispatchable DG units composed by photovoltaic panels (PVs). The power injections of PVs are represented by voltage-independent active power injections with null reactive component. It is assumed that the non-dispatchable PV injections are connected to nodes 3, 12, $22,33,45,63,76,93,109$ and 122 with the total rated power of 0.6 p.u. (base power equal to $5 \mathrm{MW}$ ). The network loads are considered as voltage independent PQ absorptions. Five DESS's are optimally placed in the system as it is shown in Fig. 3 in nodes 6, 33, 71, 100, and 123 (e.g., using [5]). It is assumed that the initial $S o C$ of all the DESSs are the same and equal to 0.3 p.u. (base energy equal to $5 \mathrm{MWh}$ ). Also, the limits for the DESS SoC are considered equal to 0.2 and 0.8 p.u.

\section{A. Clustering}

It is expected that the normal variations in supply and demand modify the borders of the zones. However, changing the borders accordingly (dynamic partitioning) contradicts the assumption that each zone should independently control its area without sharing any information with the neighbors. In order to avoid such a drawback, and establish static partitions, we ran a Probabilistic Load Flow (PLF) (e.g., [8]) instead of a classical load flow. The PLF has been performed assuming that the timevarying statistical distributions of loads and generations are normal, known and IID (for sake of space we do not present the details of the PLF process). The zones decomposition is shown in Fig. 3.

In order to validate the proposed method for the network decomposition, we have performed successive load flow calculations (each 15 minutes) using the above-mentioned statistical load/generation distributions. The partitioning is performed for each load flow run. Then, for each node, the percentage rate to belong to each area is determined. This information is shown in Fig. 4. It can be seen that every node belongs to only one area with a percentage rate of more than $85 \%$.

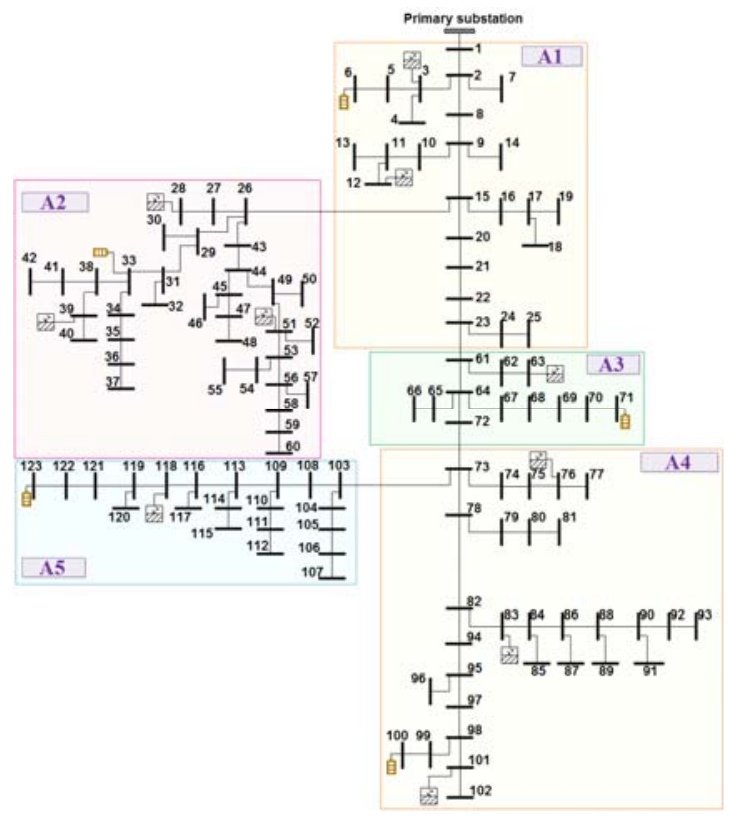

Figure 3. Clustered IEEE 123 nodes test system.

\section{B. Distributed control}

As a consequence of the network partitioning, buses 23, 26, 72,73 , are considered as CBBs. In order to validate the proposed computation of network equivalents, Fig. 5 shows the $\mathrm{CBB}$ voltage profiles along a given day with no DESS control. In particular, Fig. 5 compares the $\mathrm{CBB}$ voltage magnitudes computed by a fully-centralized load flow vs a local decentralized load flow accounting for the network equivalents. This figure shows the proper computation of the network equivalents.

In order to infer the benefits of the proposed decentralized voltage control strategy, the optimization problem mentioned in 
section III is solved using YALMIP optimizer [9]. Fig. 6 shows, for each 15-minutes period of the day, the maximum, minimum and mean values of all the network node voltages for the following cases: i) no control on DESSs, ii) decentralized control and iii) centralized control. As it can be seen, the decentralized control provides almost the same voltage improvements compared to the centralized control. In particular, the former always allows to limit the voltage deviations within the critical range of $+/-5 \%$. As expected, the proposed decentralized control leads to slightly worst voltage profiles in comparison with the centralized one. Indeed, the proposed decentralized control does not account for VSC associated to DESSs located in areas other than the targeted one.

\section{CONCLUSIONS}

The paper has illustrated a partitioning algorithm of ADNs for their distributed voltage control. The proposed algorithm relies on the direct control of DESSs and on the use of voltage sensitivity coefficients. The algorithm has been designed so that the only exchanged information between the clusters is the states of the nodes located at the boundaries of adjacent areas. The software implementation of the proposed decentralized control needs two tasks. The first one is in charge to control the areas locally, independently and in a parallel manner. This task also defines the voltage reference to be assigned to each slack node within each cluster. The second task, called E\&C, is in charge of checking the convergence of the distributed control algorithm. As a consequence, the proposed control structure still requires a centralized computation. In this respect further investigations are currently conducted by the authors to provide a more robust solution.

The effectiveness of the proposed algorithm has been verified using a test case based on the IEEE 123 buses test distribution feeder. In particular, this benchmark feeder has been modified in order to account for the presence of stochastic generation and controllable DESSs. The results have shown the good compliance of the proposed decentralized control with the corresponding, and equivalent, centralized one.

The major advantages of the proposed decentralized control can be summarized as follows: (i) remove the centralization of network information/controls, (ii) inherently reduce computation burden for the voltage optimal control problem, (iii) increase the control robustness since the proposed approach can afford to loose the voltage regulation of a given area keeping the voltages safe in others.

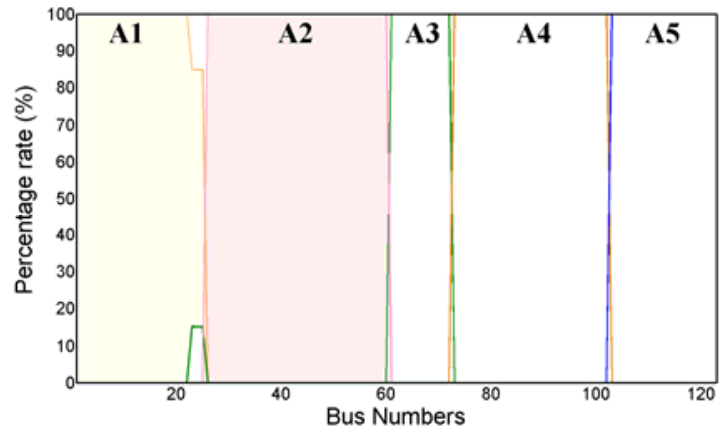

Figure 4. Percentage rate of each node to belong to a given area.
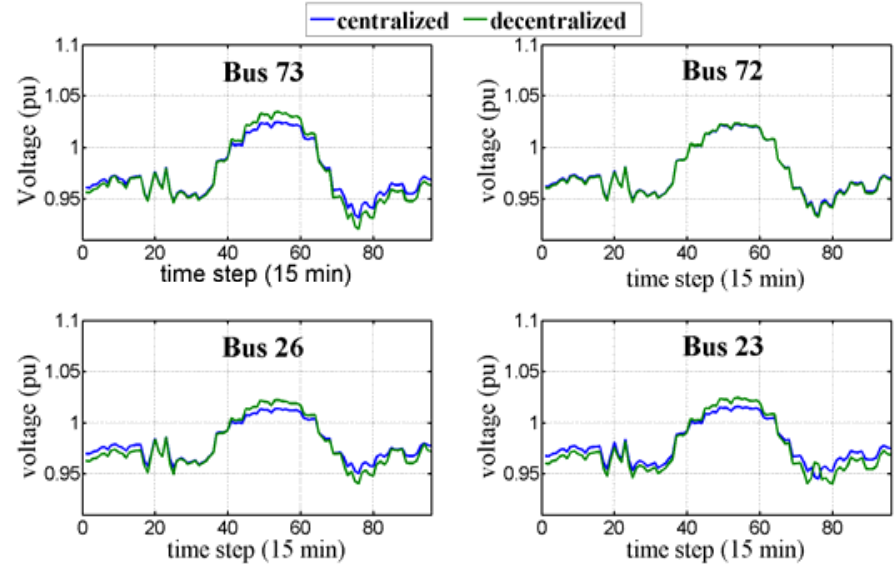

Figure 5. CBB voltage profiles (with no DESS control actions): comparison between a fully-centralized load flow and a local load flow with Thévenin equivalents (24 hours).
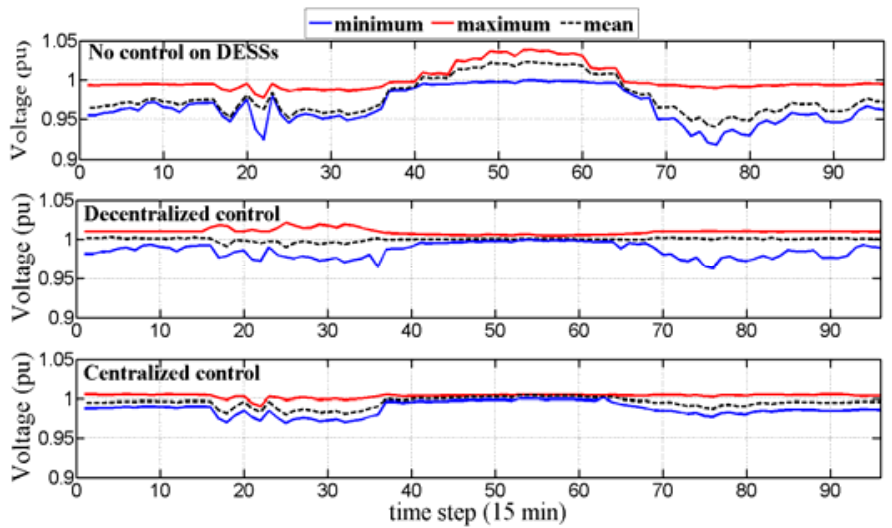

Figure 6. Distribution of all node voltages for i) no control, ii) decentralized control and iii) centralized control.

\section{REFERENCES}

[1] B. Millar, D. Jiang, and M. E. Haque, "A novel partitioning strategy for distribution networks featuring many small scale generators," IEEE PES Innovative Smart Grid Technologies Conference (ISGT), pp.1-6, 2013.

[2] P. F. Lyons, P.C. Taylor, L.M Cipcigan, P. Trichakis, and A. Wilson, "Small Scale Energy Zones and the Impacts of High Concentrations of Small Scale Embedded Generators," International Universities Power Engineering Conference, .UPEC '06, no. 2, pp. 128 - 132, 2006.

[3] T. Wang, P. Zou, and H. Zhou, "A Novel Partitioning Algorithm for Power Grid Using Community Detection," Advanced Materials Research, vol. 710, pp. 563-566, Jun. 2013.

[4] S. Mei, X. Zhang, and M. Cao, Power Grid Complexity, Springer. Tsinghua university press, 2011.

[5] M. Nick, R. Cherkaoui and M. Paolone, "Optimal Allocation of Dispersed Energy Storage Systems in Active Distribution Networks for Energy Balance and Grid Support," accepted for publication in IEEE Trans. on Power Systems, 2014.

[6] A. J. Wood and B. F. Wollenberg, power generation operation and control, New York Wiley, vol. 2, 1996.

[7] K. Christakou, J.-Y. Le Boudec, M. Paolone, and D.-C. Tomozei, "Efficient Computation of Sensitivity Coefficients of Node Voltages and Line Currents in Unbalanced Radial Electrical Distribution Networks," IEEE Trans. Smart Grid, vol. 4, no. 2, pp. 741 - 750, 2012.

[8] B. Borkowska, "Probabilistic Load Flow," IEEE Trans. Power Appar. Syst., vol. PAS-93, no. 3, pp. 752 - 759, 1973.

[9] J. Efberg, "YALMIP : A toolbox for modeling and optimization in MATLAB," IEEE International Symposium on Computer Aided Control Systems Design, pp. 284-289, 2004. 